\title{
Pós compósitos de alumina-boreto obtidos por moagem reativa de alta energia
}

\section{(Alumina-boride composite powders obtained by reactive milling)}

\author{
V. R. Cerqueira ${ }^{1,2}$, J. J. Pierri' ${ }^{2}$ M. R. Morelli ${ }^{2}$, E. M. J. A. Pallone ${ }^{3}$, A. A. M. Macêdo , R. Tomasi ${ }^{2}$ \\ ${ }^{I}$ Departamento de Ensino Técnico, Instituto Federal de Educação, Ciência e Tecnologia do Maranhão, \\ Campus Imperatriz, MA, Brasil \\ ${ }^{2}$ Departamento de Engenharia de Materiais, Universidade Federal de S.Carlos, S. Carlos, SP, Brasil \\ ${ }^{3}$ Faculdade de Zootecnia e Engenharia de Alimentos, Universidade de S. Paulo, Campus Pirassununga, SP, Brasil \\ ${ }^{4}$ IFCE Campus Quixadá, CE / Programa de Pós-Graduação em Ciência dos Materiais, \\ Universidade Federal do Maranhão, Campus Imperatriz, MA, Brasil
}

\begin{abstract}
Resumo
Este trabalho teve como objetivo sintetizar e caracterizar pós de alumina-boretos, por moagem reativa, visando a obtenção de compósitos de misturas de fases de alta dureza. Os experimentos envolveram reações de aluminotermia ativadas por moagem de alta energia, tendo como precursores $\mathrm{WO}_{3}, \mathrm{TiO}_{2}$ ou Ti, $\mathrm{B}_{2} \mathrm{O}_{3}$ ou $\mathrm{B}$ e Al. O alumínio metálico atua como agente redutor para obter misturas de alumina com boretos de titânio e de tungstênio. As reações obtidas foram do tipo auto-propagante, com tempos curtos de moagem até a ignição (2 a $15 \mathrm{~min}$ ). O tempo de ignição das reações foi monitorado por infravermelho e os produtos das reações foram mantidos sob moagem para obter partículas nanométricas. Os pós obtidos foram caracterizados por difração de raios $\mathrm{X}$ e determinação da densidade real e prensados para obter corpos de prova em forma de discos após sinterização a $1700{ }^{\circ} \mathrm{C}$ sob alto vácuo. Esses foram caracterizados por densidade aparente, difração de raios X e microscopia eletrônica de varredura. Os pós resultantes da moagem de alta energia mostraram completa transformação dos reagentes, formando pós com composições do sistema $\mathrm{Al}_{2} \mathrm{O}_{3}-\left(\mathrm{WB} / \mathrm{W}_{2} \mathrm{~B} / \mathrm{WB}_{2} / \mathrm{W}_{2} \mathrm{~B}_{5}\right)-\mathrm{TiB}_{2}$. Dependendo da reação de síntese, os pós obtidos apresentaram diferentes comportamentos durante a sinterização: pós que densificaram até porosidade aparente próxima de zero e pós que não densificaram, resultando em porosidade aparente em torno de $30 \%$.

Palavras-chave: moagem de alta energia; cerâmica de alta dureza, boretos, compósito.
\end{abstract}

\begin{abstract}
This study aimed to synthesize and characterize alumina-boride powders by reactive milling to obtain composite of mixtures of high hardness phases. The experiment involved aluminothermic reactions activated by high energy milling of $\mathrm{WO}_{3}$, TiO $\mathrm{O}_{2}$, or $\mathrm{Ti}$, $\mathrm{B}_{2} \mathrm{O}_{3}$ or $\mathrm{B}$ and aluminum powder, as precursors. Aluminum is the reducing agent to obtain a mixing of alumina with titanium and tungsten borides. The reactions were of the self-propagating type, with short milling times until the ignition (between 2-15 min). The ignition time of the reactions was monitored by infrared and the reaction products were kept under additional milling time to obtain nanometric particles. The powders were characterized by X-ray diffraction and measurement of density. Specimens pressed in form of discs and sintered at $1700^{\circ} \mathrm{C}$ under high vacuum were characterized by apparent density, X-ray diffraction and scanning electron microscopy. The results showed complete conversion of the reactants forming multiphase powders of the system $\mathrm{Al}_{2} \mathrm{O}_{3}-(\mathrm{WB} /$ $\left.W_{2} B / W B_{2} / W_{2} B_{5}\right)-T i B_{2}$. Depending on the synthesis reaction, the powder presented different behavior during sintering: powders that densify to approximately zero porosity and powders that do not densify, with final apparent porosity of about $30 \%$.
\end{abstract}

Keywords: high energy ball milling; ceramic high hardness, borides, composite.

\section{INTRODUÇÃ̃O}

Estudos experimentais mostram que a moagem de alta energia (MAE) é um método viável para obtenção de pós de compostos e misturas cerâmica-cerâmica, cerâmicametal (intermetálico), incluindo óxidos metal-intermétalicos, carbetos, silicetos, nitretos e boretos [1-6]. Na MAE podem ocorrer reações autossustentadas induzidas pela moagem de modo similar às reações de síntese autopropagante a altas temperaturas, denominadas reações SHS (self-propaganting high-temperature synthesis) [6]. Essas reações são ativadas pelo aquecimento da mistura de pós reagentes a uma temperatura de ignição. $\mathrm{O}$ tipo de reação e as condições de moagem requer certo tempo de moagem para iniciar a reação. A partir da temperatura de ignição a reação é exotérmica e se propaga por todo o volume de reagente [6]. Na moagem de alta energia com reação do tipo aluminotérmica, o alumínio reduz óxidos de diferentes metais possibilitando a obtenção de reações em curtos tempos de moagem [7-9], liberando o calor excedente e permite a formação, quando na presença dos reagentes adequados, de boretos, carbetos, nitretos dos metais reduzidos. Dentre os produtos de reação encontra- 
se alto teor de alumina. A eliminação desta por processos físico-químicos é possível [10], todavia podem-se incluir processos que atacam os compostos de interesse. Por outro lado, a presença da alumina nos produtos da reação também possibilita a obtenção de compósitos com alta dureza, resistência mecânica a flexão e ao desgaste, podendo ainda resultar em aumento de tenacidade, em compósitos de matriz de alumina com baixos teores de inclusões nanométricas de fases de alta dureza, dentre as quais se incluem $\mathrm{NbC}$ [3], TiC [11], $\mathrm{WC}$ [12], $\mathrm{Ni}_{3} \mathrm{Al}$ [13]. O presente trabalho apresenta os resultados da síntese por moagem com reação aluminotérmica de pós de misturas do sistema $\mathrm{Al}_{2} \mathrm{O}_{3}-\left(\mathrm{WB} / \mathrm{W}_{2} \mathrm{~B} / \mathrm{WB}_{2} / \mathrm{W}_{2} \mathrm{~B}_{5}\right)$ - $\mathrm{TiB}_{2}$, bem como a verificação da viabilidade de sinterização desses pós. $\mathrm{O}$ interesse neste sistema decorre não apenas da possibilidade de obter compósitos de alumina com fases dispersas de alta dureza, mas também pela possibilidade de formação de diversas fases de boretos de tungstênio e pela solubilidade desses no boreto de titânio, a altas temperaturas $[14,15]$.

\section{MATERIAIS E METODOS}

Reações altamente exotérmicas foram obtidas por moagem em moinho de alta energia (Spex 8000 Mixer Mill) dos reagentes (precursores) comerciais: óxido de titânio $\left(\mathrm{TiO}_{2}\right)$-Vetec, óxido de boro $\left(\mathrm{B}_{2} \mathrm{O}_{3}\right)$ - Aldrich, óxido de tungstênio $\left(\mathrm{WO}_{3}\right)$ - Alfa Aesar, titânio metálico em pó $\left(\mathrm{Ti}^{\circ}\right)$ Alfa Aesar, boro metálico $\left(\mathrm{B}^{\circ}\right)$ - Aldrich e alumínio metálico como redutor. Os parâmetros utilizados para a moagem de alta energia foram estabelecidos mediante trabalhos já publicados [11-13]. A relação massa de bolas / massa de material foi 4:1. O frasco do moinho e as bolas utilizadas foram de aço endurecido por tratamento térmico, sendo o volume útil do moinho e diâmetro das bolas de $150 \mathrm{~mL}$ e 11 $\mathrm{mm}$, respectivamente. As proporções molares dos reagentes foram calculadas para se obter produtos constituídos de alumina $\left(\mathrm{Al}_{2} \mathrm{O}_{3}\right)$ com diferentes teores de boretos de titânio $\left(\mathrm{TiB}_{2}\right)$ e de tungstênio (WB, $\mathrm{WB}_{2}$ e $\mathrm{W}_{2} \mathrm{~B}_{5}$ ). A inserção de $\mathrm{Ti}^{\circ}$ ou de $\mathrm{B}^{\circ}$ produz maiores proporções de $\mathrm{TiB}_{2}$. As reações químicas (A, B, C, D e E) realizadas são apresentadas abaixo, bem como, os valores calculados das porcentagens em massa dos produtos, na mesma ordem em que aparecem na reação:

$$
\begin{aligned}
3 \mathrm{TiO}_{2}+10 \mathrm{~B}_{2} \mathrm{O}_{3}+7 \mathrm{WO}_{3}+38 \mathrm{Al} \rightarrow & 19 \mathrm{Al}_{2} \mathrm{O}_{3}+7 \mathbf{W B}_{2}+3 \mathrm{TiB}_{2}(\mathrm{~A}) \\
& (54,1-40,1-5,8) \% \text { massa } \\
3 \mathrm{Ti}+4 \mathrm{~B}_{2} \mathrm{O}_{3}+\mathrm{WO}_{3}+10 \mathrm{Al} \rightarrow 5 & \mathrm{Al}_{2} \mathrm{O}_{3}+\mathbf{W B}_{2}+3 \mathrm{TiB}_{2} \quad(\mathrm{~B}) \\
& (55,2-22,2-22,6) \% \text { massa } \\
3 \mathrm{TiO}_{2}+8 \mathbf{B}+\mathrm{WO}_{3}+6 \mathrm{Al} \rightarrow 3 \mathrm{Al}_{2} \mathrm{O}_{3}+\mathbf{W B}_{2}+3 \mathrm{TiB}_{2} \quad(\mathrm{C}) & (42,5-28,5-29,0) \% \text { massa } \\
3 \mathrm{TiO}_{2}+8 \mathrm{~B}_{2} \mathrm{O}_{3}+10 \mathrm{WO}_{3}+40 \mathrm{Al} \rightarrow & 20 \mathrm{Al}_{2} \mathrm{O}_{3}+10 \mathbf{W B}+3 \mathrm{TiB}_{2}(\mathrm{D}) \\
& (48,6-46,4-5,0) \% \text { massa } \\
3 \mathrm{TiO}_{2}+13 \mathrm{~B}_{2} \mathrm{O}_{3}+8 \mathrm{WO}_{3}+46 \mathrm{Al} & \rightarrow 23 \mathrm{Al}_{2} \mathrm{O}_{3}+4 \mathbf{W}_{2} \mathbf{B}_{5}+3 \mathrm{TiB}_{2}(\mathrm{E}) \\
& (55,4-39,7-4,9) \% \text { massa })
\end{aligned}
$$

Para a determinação do tempo de ignição das reações, o momento da ignição foi monitorado pela ocorrência de um pico de temperatura na superfície externa do frasco de moagem. Para isso, foi utilizado um sensor térmico de infravermelho Raytek-Thermalet MID, posicionado $10 \mathrm{~cm}$ distante do frasco em movimento. Após reação, os produtos foram mantidos sob moagem, por tempos diferentes, visando a obtenção de partículas nanométricas $\mathrm{e}$, posteriormente, desaglomerados em meio de álcool anidro, em moinho de bolas e planetário, utilizando-se bolas de zircônia e aço temperado, respectivamente. Durante a desaglomeração, em moinho de bolas, foram adicionados ácido para-aminobenzoico (PABA) e ácido oleico, 0,2 e $0,5 \%$, respectivamente, como dispersantes e lubrificantes para auxiliar a conformação por prensagem. Os pós obtidos foram caracterizados por difração de raios X por meio de um difratômetro da marca Siemens modelo 5100, com radiação $\mathrm{k} \alpha(\mathrm{Cu})$ e tensão e corrente, respectivamente, de $40 \mathrm{kV}$ e $40 \mathrm{~mA}$. Os pós foram conformados na forma de pastilhas cilíndricas de $10 \mathrm{~mm}$ de diâmetro e $\sim 3 \mathrm{~mm}$ de altura por prensagem uniaxial a $60 \mathrm{MPa}$, e sinterizados a $1700{ }^{\circ} \mathrm{C}$ por $1 \mathrm{~h}$, sob alto vácuo $\left(\sim 10^{-5} \mathrm{mbar}\right)$, em forno Thermal Technology Inc. com elemento resistivo de tungstênio. As amostras sinterizadas foram caracterizadas por difração de raios X (difratômetro Siemens 5100) e microscopia eletrônica de varredura (MEV) Philips XL30-FEG e por densidade aparente pelo método de Arquimedes.

\section{RESULTADOS E DISCUSSÃO}

A Fig. 1 apresenta curvas de variação da temperatura na superfície dos frascos de moagem com o tempo, durante o processamento de síntese, obtidas para cada uma das reações consideradas. Observa-se o pico decorrente da reação abrupta, típica de uma reação auto-propagante. O início do pico é bem definido e o tempo de moagem necessário para que ocorra é tomado como o tempo para ignição $\left(t_{\mathrm{ig}}\right)$, para condições experimentais mantidas constantes, pode ser

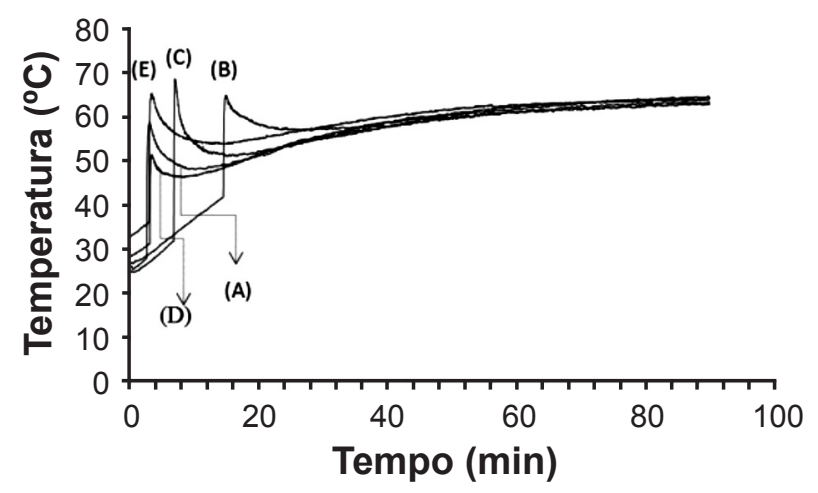

Figura 1: Variação da temperatura externa do moinho durante a moagem em função do tempo para as reações (A), (B), (C), (D) e (E).

[Figure 1: External temperature variation of the mill during milling as a function of time for the reactions (A), (B), (C), (D) and (E).] 
associado às características dos pós reagentes, em particular a pressão parcial de oxigênio de equilíbrio das reações de redução envolvidas, bem como, as características físicas dos pós reagentes que são determinantes no comportamento destes durante o processo de moagem.

$\mathrm{Na}$ Tabela I são apresentados os tempos de ignição $\left(\mathrm{t}_{\mathrm{ig}}\right)$ para as diferentes reações. Nota-se que esses tempos são relativamente curtos, quando comparados aos tempos de moagem típicos na moagem reativa. Também é evidente a diferença entre os $t_{i g}$ das reações que envolvem apenas reagentes óxidos e as que incluem o Ti ou o $\mathrm{B}$. Considerando a sequência de reações parciais previstas para os casos em estudo, as reações iniciais e que participam diretamente da ignição são reações de redução e, portanto, não seria esperado um efeito significativo dos reagentes metálicos no $t_{\mathrm{ig}}$. A redução do $\mathrm{WO}_{3}$ é a que apresenta maior pressão parcial de oxigênio de equilíbrio sendo, portanto, a primeira a ocorrer e responsável pela ignição. Por outro lado, as quantidades de $\mathrm{WO}_{3}$ entre os reagentes das reações envolvendo apenas reagentes óxidos são significativamente superiores às quantidades de $\mathrm{WO}_{3}$ entre os reagentes das reações envolvendo reagentes metálicos. A Fig. 2 mostra a forte relação entre $t_{\mathrm{ig}}$ e a quantidade de $\mathrm{WO}_{3}$. Os reagentes metálicos participam das reações de formação dos boretos, nas altas temperaturas que são rapidamente atingidas após a ignição, como demonstram, considerando o caráter autopropagante das reações, os altos valores das temperaturas adiabáticas calculadas para as mesmas, também apresentadas na Tabela I.

A Fig. 3 apresenta os difratogramas de raios $\mathrm{X}$ dos produtos das reações. A ocorrência da reação é confirmada pela ausência de fases reagentes. No entanto, nos produtos das reações, são identificadas misturas de fases de boreto de tungstênio, combinando a fase correspondente à relação $\mathrm{W}: \mathrm{B}$ dada pela estequiometria da reação com outras fases de boreto de tungstênio contendo menor proporção de boro, conforme mostra a Tabela II. Isso pode ser um indicativo de que as reações ocorridas não atingiram o equilíbrio e a completa homogeneização, considerando a velocidade em que ocorrem assim como a complexidade do diagrama de fases W-B, nas altas temperaturas que devem ser atingidas para que ocorram as reações autopropagantes obtidas. Observa-se também, a ausência de picos de difração correspondentes ao $\mathrm{TiB}_{2}$ na amostra obtida pela reação $\mathrm{D}$. Nessa reação, a porcentagem em massa de $\mathrm{TiB}_{2}$ que pode ser esperada, considerando a estequiometria, em torno de 5\%, é muito próxima às das reações $\mathrm{A} e \mathrm{E}$. Para estas, no entanto, os picos de difração do $\mathrm{TiB}_{2}$ estão presentes. A principal diferença da reação $\mathrm{D}$ em relação às outras é a maior proporção de tungstênio em relação ao boro, contendo maior quantidade relativa de óxido de tungstênio como reagente. Uma decorrência disso é a maior temperatura adiabática dessa reação. A Fig. 4 mostra o diagrama de equilíbrio de fases do sistema $\mathrm{TiB}_{2}-\mathrm{WB}_{2}$ que, na extremidade esquerda, de baixos teores de $\mathrm{TiB}_{2}$, indica o aumento da solubilidade deste no $\mathrm{WB}_{2}$ com o aumento da temperatura, bem como a formação da fase líquida a uma temperatura eutética de aproximadamente $2250{ }^{\circ} \mathrm{C}$. Considerando a maior temperatura adiabática da reação, a formação de solução sólida e mesmo a formação de fase líquida e o resfriamento rápido dos produtos da reação durante a moagem pode explicar a ausência de picos de $\mathrm{TiB}_{2}$ no difratograma.

Tabela I - Tempos para Ignição e densidades real dos pós obtidos e valores calculados da densidade e das temperaturas adiabáticas para as diferentes reações.

[Table I - Times Ignition and real densities of the powders and calculated values of the density and adiabatic temperatures for the different reactions.]

\begin{tabular}{|c|c|c|c|c|c|c|}
\hline & $\begin{array}{c}\text { Reações } \\
(\% \text { em massa de reagentes) }\end{array}$ & $\begin{array}{c}\text { Tempo para } \\
\text { ignição }\left(\mathrm{t}_{\mathrm{ig}}\right) \\
\quad *(\mathrm{~min})\end{array}$ & $\begin{array}{c}\text { Densidade } \\
\text { real } \\
(\mathrm{dr})^{* *} \\
\left(\mathrm{~g} / \mathrm{cm}^{3}\right)\end{array}$ & $\begin{array}{c}\text { Densidade } \\
\text { calculada } \\
(\mathrm{dc})^{* * *} \\
\left(\mathrm{~g} / \mathrm{cm}^{3}\right)\end{array}$ & $\begin{array}{l}\text { Diferença } \\
\text { entre dr e dc } \\
\quad\left(\mathrm{g} / \mathrm{cm}^{3}\right)\end{array}$ & $\begin{array}{c}\text { Temperatura } \\
\text { adiabática } \\
\left({ }^{\circ} \mathrm{C}\right)\end{array}$ \\
\hline (A) & $\begin{array}{c}\left(3 \mathrm{TiO}_{2}+10 \mathrm{~B}_{2} \mathrm{O}_{3}+7 \mathrm{WO}_{3}+38 \mathrm{Al}\right) \\
(6,6-19,3-45,7-28,4)\end{array}$ & 2,58 & 5,27 & 5,65 & 0,38 & 2617 \\
\hline (B) & $\begin{array}{r}\left(3 \mathrm{Ti}+4 \mathrm{~B}_{2} \mathrm{O}_{3}+\mathrm{WO}_{3}+10 \mathrm{Al}\right) \\
\quad(15,5-30,0-25,4-29,1)\end{array}$ & 14,52 & 4,69 & 4,9 & 0,21 & 2773 \\
\hline (C) & $\begin{array}{l}\left(3 \mathrm{TiO}_{2}+8 \mathrm{~B}+\mathrm{WO}_{3}+6 \mathrm{Al}\right) \\
\quad(33,1-11,9-32,6-22,4)\end{array}$ & 6,83 & 4,91 & 5,24 & 0,33 & 2617 \\
\hline (D) & $\begin{array}{c}\left(3 \mathrm{TiO}_{2}+8 \mathrm{~B}_{2} \mathrm{O}_{3}+10 \mathrm{WO}_{3}+40 \mathrm{Al}\right) \\
(5,7-13,2-55,7-25,5)\end{array}$ & 3,15 & 5,76 & 6,12 & 0,36 & 3205 \\
\hline (E) & $\begin{array}{c}\left(3 \mathrm{TiO}_{2}+13 \mathrm{~B}_{2} \mathrm{O}_{3}+8 \mathrm{WO}_{3}+46 \mathrm{Al}\right) \\
\quad(5,6-21,2-44,2-29,0)\end{array}$ & 2,23 & 5,16 & 5,47 & 0,31 & 2861 \\
\hline
\end{tabular}

* tempo determinado em um único experimento de moagem; ** obtido por picnômetro a hélio Accupyo 1330, Micromeritics; *** composição do produto é dada pelas reações utilizadas para a definição das proporções de reagentes. 


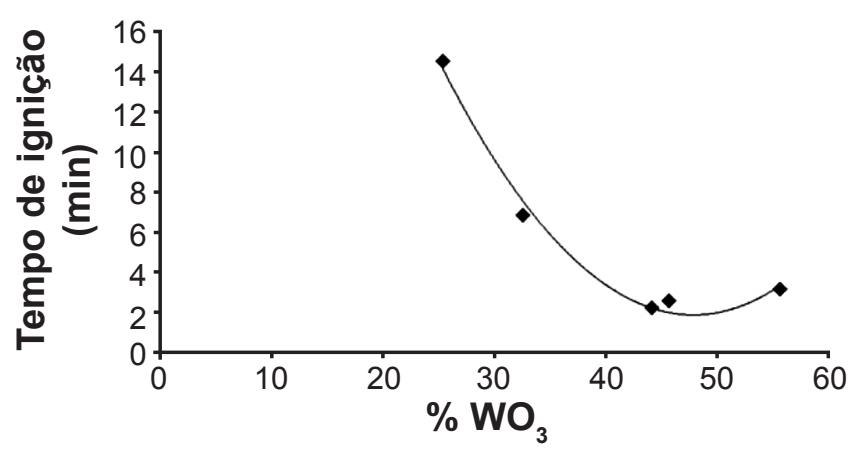

Figura 2: Variação do tempo de moagem para a ocorrência da ignição como função da porcentagem em massa de $\mathrm{WO}_{3}$ na mistura de reagentes.

[Figure 2: Grinding time variation of the occurrence of the ignition as a function of the percentage by mass of $\mathrm{WO}_{3}$ in the mixture of reagents.]

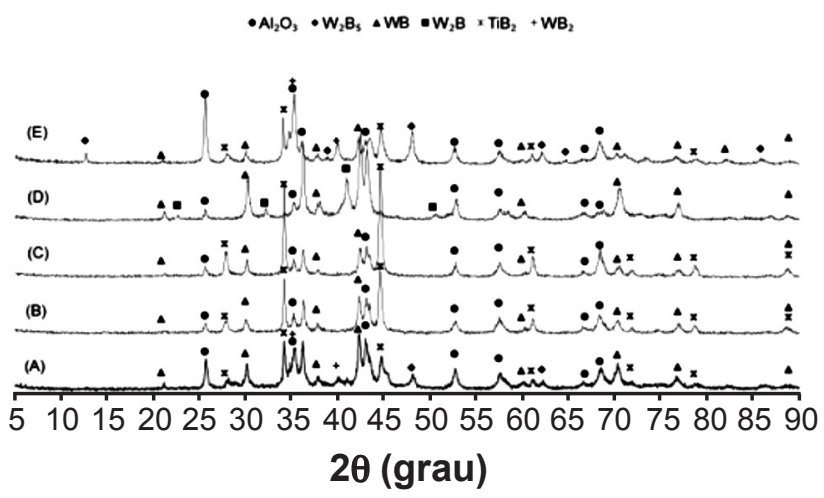

Figura 3: Difratogramas de raios $\mathrm{X}$ dos pós obtidos a partir das reações $\mathrm{A}, \mathrm{B}, \mathrm{C}, \mathrm{D}$ e E por moagem reativa.

[Figure 3: XRD patterns of the powders obtained from the reactions $A, B, C, D$ and $E$ by reactive grinding.]

A Tabela III apresenta os resultados obtidos com a sinterização a $1700^{\circ} \mathrm{C}$, incluindo: a perda de massa percentual dos corpos de prova durante a sinterização e a porosidade e densidade aparentes. Destacam-se, em particular a grande diferença entre os resultados de baixa porosidade para as amostras obtidas a partir das reações B, C e D com relação às das reações $\mathrm{A}$ e $\mathrm{E}$ que tiveram as maiores perdas de massa e apresentaram alta porosidade aparente e baixa densidade aparente. A alta perda de massa durante a sinterização pode ser devida à evaporação do boro e também do tungstênio, levando-se em consideração as condições de alto vácuo e de alta temperatura de sinterização. Uma indicação da perda de boro é dada pelos resultados de DRX mostrados na Fig. 5 , em que se observa a presença de fases com relação $B / W$ menor que a fase esperada pela estequiometria das reações. Destaca-se também a diferença de comportamento durante a sinterização entre as amostras A e E assim como, as demais. Todas apresentam altas perdas de massa, porém nas reações A e E destaca-se a manutenção da alta porosidade do corpo não sinterizado, enquanto em B, C e D, a densificação é acompanhada de grande retração dos corpos de prova.

A Fig. 5 apresenta as curvas de difração de raios $\mathrm{X}$ das amostras obtidas após sinterização a $1700{ }^{\circ} \mathrm{C}$, com os pós produzidos pelas reações B, C e D. A alumina está
Tabela II - Boretos de tungstênio calculados e formados a partir das reações A, B, C, D e E por moagem reativa. [Table II - Tungsten borides calculated and formed from the reactions $A, B, C, D$ and $E$ by reactive milling.]

\begin{tabular}{ccc}
\hline Reação & $\begin{array}{c}\text { Fase } \mathrm{W}_{\mathrm{x}} \mathrm{B}_{\mathrm{y}} \text { dada } \\
\text { pela reação }\end{array}$ & $\begin{array}{c}\text { Fase } \mathrm{W}_{\mathrm{x}} \mathrm{B}_{\mathrm{y}} \\
\text { observada por DRX }\end{array}$ \\
\hline (A) & $\mathrm{WB}_{2}$ & $\mathrm{WB}, \mathrm{WB}_{2} / \mathrm{W}_{2} \mathrm{~B}_{5}$, \\
$(B)$ & $\mathrm{WB}_{2}$ & $\mathrm{WB}$ \\
$($ C) & $\mathrm{WB}_{2}$ & $\mathrm{WB}$ \\
$(\mathrm{D})$ & $\mathrm{WB}$ & $\mathrm{WB}$ \\
(E) & $\mathrm{W}_{2} \mathrm{~B}_{5}$ & $\mathrm{WB}, \mathrm{WB}_{2}, \mathrm{~W}_{2} \mathrm{~B}_{5}$ \\
\hline
\end{tabular}

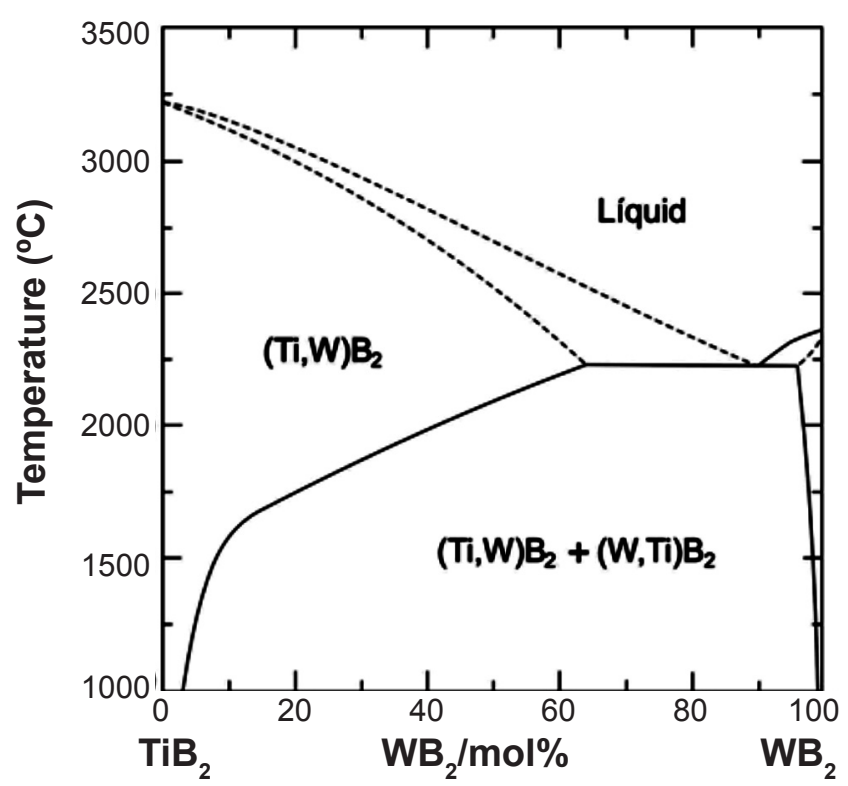

Figura 4: Diagrama de equilíbrio das fases $\mathrm{TiB}_{2}$ e $\mathrm{WB}_{2}[15]$.

[Figure 4: Equilibrium diagram of $\mathrm{TiB}_{2}$ and $\mathrm{WB}_{2}$ phases [15]].

Tabela III - Densidade aparente, porosidade aparente e perda de massa dos compósitos obtidos a partir das reações A, B, $\mathrm{C}, \mathrm{D}$ e E e sinterizados a $1700^{\circ} \mathrm{C}$

[Table III - Apparent density, apparent porosity and mass loss of the composites obtained from the reactions $A, B, C, D$ and $E$ and sintered at $1700^{\circ} \mathrm{C}$.]

\begin{tabular}{cccc}
\hline Reação & $\begin{array}{c}\text { Perda de } \\
\text { massa } \\
(\%)\end{array}$ & $\begin{array}{c}\text { Porosidade } \\
\text { aparente } \\
(\%)\end{array}$ & $\begin{array}{c}\text { Densidade } \\
\text { aparente } \\
\left(\mathrm{g} / \mathrm{cm}^{3}\right)\end{array}$ \\
\hline (A) & 16,0 & 34,0 & 3,6 \\
(B) & 11,1 & 0,8 & 4,5 \\
(C) & 15,5 & 0,8 & 4,8 \\
(D) & 14,6 & 0,0 & 5,9 \\
(E) & 18,3 & 28,2 & 3,8 \\
\hline
\end{tabular}

presente em todas as amostras confirmando sua condição de fase predominante e mais estável nos produtos de todas as reações. Para as amostras obtidas a partir das reações B e 


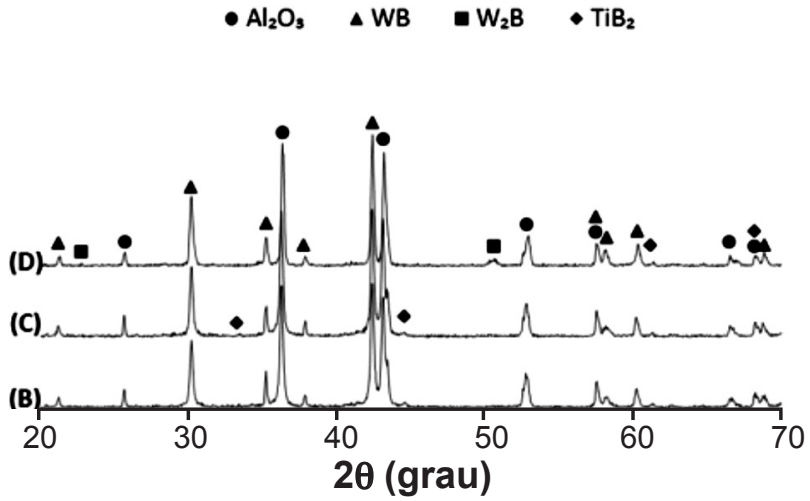

Figura 5: Difratogramas de raios $\mathrm{X}$ dos compósitos obtidos a partir das reações $\mathrm{B}, \mathrm{C}$ e D e sinterizados $1700{ }^{\circ} \mathrm{C}$.

[Figure 5: XRD patterns of the composites obtained from the reactions $B, C$ and $D$ and sintered $\left.1700^{\circ} \mathrm{C}\right]$
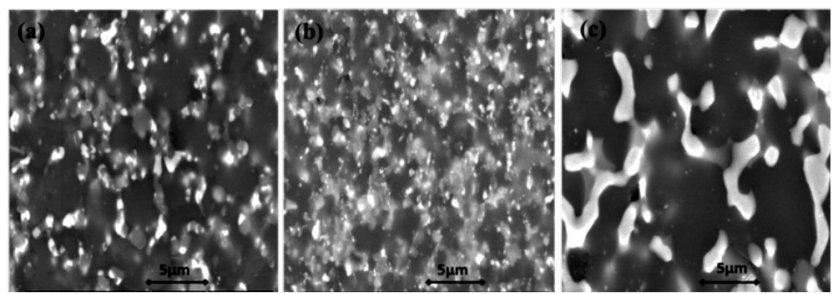

Figura 6: Micrografias obtidas por microscopia eletrônica de varredura da superfície polida dos compósitos sinterizados 1700 ${ }^{\circ} \mathrm{C}$ obtidos a partir das reações B-(a), C-(b) e D-(c).

[Figure 6: SEM images of polished surface of the composites sintered at $1700{ }^{\circ} \mathrm{C}$ obtained from the reactions $B-(a), C-(b)$ and $D-(c)]$.

$\mathrm{C}$, a maior presença de $\mathrm{TiB}_{2}$ resulta em picos identificados nos difratogramas, enquanto na amostra sinterizada oriunda da reação D não é observado. Como discutido acima, nessa última o teor de $\mathrm{TiB}_{2}$ previsto pela reação é baixo e pode ser inferior ao limite de detecção da difração de raiox $\mathrm{X}$ ou pode ter ocorrido a solubilização nas fases ricas em boretos de tungstênio.

Com relação aos boretos de tungstênio, o WB é a única fase observada nas amostras sinterizadas com os pós das reações $\mathrm{B}$ e $\mathrm{C}$ e é predominante na obtida a partir da reação $\mathrm{D}$, na qual é identificada a presença de $\mathrm{W}_{2} \mathrm{~B}$. Este último resultado é mais uma evidência de que a perda de massa, mais alta nessa amostra, está associada à perda de boro. De qualquer modo, em todos os casos a relação B:W deve estar menor do que a dada pela estequiometria das reações. Essa variação de composição pode ser explicada pela perda de massa acima relatada, ocorrendo provavelmente uma perda maior do boro com relação ao tungstênio. A mistura de fases e a possível presença de soluções sólidas dificultam a determinação quantitativa das fases presentes nas amostras sinterizadas e o cálculo da densidade teórica das mesmas. Assim, também não é possível fazer uma comparação entre os valores de densidade aparente apresentados na Tabela III. Grosseiramente, considerando que as reações de síntese são similares, é significativa a diferença de resultados entre as reações $\mathrm{A}$ e $\mathrm{E}$, que resultaram em pós que não densificaram durante a sinterização e as demais reações que produziram amostras densas, com porosidade aparente próxima de zero.

A Fig. 6 apresenta as micrografias obtidas por MEV da superfície polida das amostras sinterizadas a $1700{ }^{\circ} \mathrm{C}$, produzidas com os pós resultantes das reações $\mathrm{B}, \mathrm{C}$ e $\mathrm{D}$. As regiões escuras são formadas pela alumina que aparece como fase majoritária. Os boretos de tungstênio e titânio estão nas regiões claras. Estes, de modo geral, aparecem como inclusões de formas arredondadas como resultado da alta energia das superfícies entre os boretos e a alumina, o que causa não apenas o arredondamento das inclusões, mas também a tendência à coalescência das mesmas, tomando as formas alongadas nos contornos de grãos da alumina. Nas amostras obtidas das reações B e C, os boretos encontramse em formas arredondadas, porém, com relação à amostra obtida da reação $\mathrm{D}$, estão mais dispersos nos contornos de grãos de alumina, os quais são menores. Nesses casos observam-se também inclusões submicrométricas de boretos nos contornos e interior dos grãos de alumina.

\section{CONCLUSÕES}

A moagem reativa do tipo aluminotérmica mostrouse eficaz para a obtenção de pós cerâmicos de misturas de fases do tipo $\mathrm{Al}_{2} \mathrm{O}_{3}-\mathrm{W}_{\mathrm{x}} \mathrm{B}_{\mathrm{y}}-\mathrm{TiB}_{2}$. As reações obtidas foram altamente exotérmicas e autopropagantes e ocorreram em curtos espaços de tempo. Devido a uma proporção B:W possivelmente menor do que o dado pela estequiometria das reações e a perda de boro durante a sinterização, os pós compósitos obtidos resultaram, após sinterização, em compósitos contendo apenas o WB, dentre as fases de boretos de tungstênio. No entanto, os pós obtidos de diferentes reações, apresentaram resultados distintos na sinterização: os que praticamente não densificaram durante a sinterização (porosidade aparente próxima de $30 \%$ ) e os que atingiram porosidade aparente próxima de zero. Este último resultado permitiu verificar que, mesmo contendo elevadas proporções de boretos, fases que formam superfícies de alta energia com a alumina, é possível a obtenção de corpos de compósitos cerâmicos de fases de alta dureza com microestruturas relativamente homogêneas e com alta densidade.

\section{REFERÊNCIAS}

[1] M. J. Mayo, Int. Mater. Rev. 41 (1996) 85.

[2] E. M. J. A. Pallone, V. Trombini, M. S. Nascimento, W. Botta, R. Tomasi, Mater. Sci. Forum 14 (2002) 65.

[3] E. M. J. A. Pallone, V. Trombini, W. J. Botta F, R. Tomasi, J. Mater. Proc. Technol. 143-144 (2003) 185.

[4] B. S. Murty, S. Ranganathan, Int. Mater. Rev. 43 (1998) 101.

[5] C. Suryanarayana, Prog. Mater. Sci. 46 (2001) 1.

[6] K. Niihara. J. Ceram. Soc. Jpn. 99 (1991) 974.

[7] V. Trombini1, A. H. A. Bressiani1, E. M. J. A. Pallone, R. Tomasi, Cerâmica 57, 344 (2011) 416.

[8] W. Botta, R. Tomasi, E.M. J. A Pallone, A. Yavari, Scripta Mater. 44 (2001) 1735.

[9] E. M. J. A. Pallone, V. Trombini, W. Botta, R. Tomasi, 
Proc. Metastable Nanostructured Mater., Nanomat-2001, 403 (2002) 65.

[10] S. Sarker, Hydrometal. 105 (2011) 364.

[11] V. Trombini, E. M. J. A Pallone, F. Mello, W. Botta, R. Tomasi, Adv. Powder Technol. III 416-418 (2003) 475.

[12] E. M. J. A Pallone, D. Martin, R. Tomasi, W. Botta,
Mater. Sci. Eng. A-Struct. Mater. Prop. 464 (2007) 47.

[13] D. Leiva, E.M. J. A Pallone, R. Tomasi, W. Botta, Adv. Powder Technol. III, 416-418 (2003)493.

[14] R. Telle, E. Fendler, G. Petzow, J. Hard Mater. 3 (1992) 211.

[15] I. Mitra, R. Telle, J. Solid State Chem. 133 (1997) 25. (Rec. 19/09/2014, Rev. 20/12/2014, Ac. 31/01/2015) 